\section{Revista Brasileira de Administração Científica}

Brazilian Journal of Scientific Administration

Abr a Jun $2021-$ v.12 - n.2

\title{
Propriedade intelectual: levantamento de patentes no setor de turismo
}

As organizações de turismo, nos últimos anos, começaram a investir cada vez mais na gestão do conhecimento, mas ainda é possível perceber a relutância de algumas organizações em empregar a inovação na gestão organizacional das empresas. O turismo no Brasil é uma atividade econômica relativamente importante, com grande potencial de crescimento futuro. Em 2018, a contribuição do turismo ao PIB brasileiro foi de US\$ 152,5 bilhões ( $8,1 \%$ ), sendo que o PIB turístico teve crescimento de 3,1\%, um dos mais elevados da América do Sul, representando o dobro do que cresceu a economia brasileira. A boa nova do aumento do gasto internacional é atribuída ao recém-criado visto eletrônico oferecido aos Estados Unidos, Canadá, Austrália e Japão. Este trabalho teve como objetivo analisar através de uma prospecção tecnológica, o depósito de patentes do setor de turismo. 0 estudo exploratório foi realizado com base nos pedidos de patente no setor de turismo depositados na plataforma World Intellectual Property Organization (OMPI), até novembro de 2019. Os principais resultados do estudo mostram que a China, Estados Unidos e a Espanha foram os países que mais depositaram patente, e que o Brasil ainda tem muito que crescer no tocante as novas tecnologias para o setor de turismo, precisando ampliar o discurso e estratégias para um turismo mais inteligente e se tornar mais competitivo no segmento.

Palavras-chave: Inovação; Tecnologia; Turismo; Prospecção.

\section{Intellectual property: lifting patents in the tourism sector}

\begin{abstract}
Tourism organizations, in recent years, have started to invest more and more in knowledge management, but it is still possible to perceive the reluctance of some organizations to employ innovation in the organizational management of companies. Tourism in Brazil is a relatively important economic activity, with great potential for future growth. In 2018, the contribution of tourism to the Brazilian GDP was US $\$ 152.5$ billion (8.1\%), with tourism GDP growing by $3.1 \%$, one of the highest in South America, representing double than the Brazilian economy grew. The good news of the increase in international spending is attributed to the newly created electronic visa offered to the United States, Canada, Australia and Japan. This work aimed to analyze, through a technological exploration, the deposit of patents in the tourism sector. The exploratory study was carried out based on patent applications in the tourism sector filed with the World Intellectual Property Organization (WIPO) platform, until November 2019. The main results of the study show that China, the United States and Spain were the countries that more filed a patent, and that Brazil still has a lot to grow with regard to new technologies for the tourism sector, needing to expand the discourse and strategies for smarter tourism and become more competitive in the segment.
\end{abstract}

Keywords: Innovation; Technology; Tourism; Prospection.

Topic: Inovação Tecnológica

Reviewed anonymously in the process of blind peer.
Received: 17/04/2021

Approved: 10/06/2021
Mônica Maria Liberato (iD

Universidade Federal de Sergipe, Brasil http://lattes.cnpq.br/6716169981657880 http://orcid.org/0000-0001-6367-0632 monicaliberato@hotmail.com

\section{Cleo Clayton Santos Silva (iD}

Universidade Federal de Sergipe, Brasil http://lattes.cnpq.br/0833666295346324

http://orcid.org/0000-0002-7595-3063 cleoclayton@hotmail.com

Nadja Rosele Alves Batista (iD)

Universidade Federal de Sergipe, Brasil

http://lattes.cnpq.br/0712177778532687

http://orcid.org/0000-0003-0932-2041

nrosele.nr@gmail.com
Francisco Sandro Rodrigues Holanda (10) Universidade Federal de Sergipe, Brasil http://lattes.cnpq.br/7935943969315043 http://orcid.org/0000-0001-7913-0585 fholanda@infonet.com.br
Referencing this:

LIBERATO, M. M.; SILVA, C. C. S.; BATISTA, N. R. A.; HOLANDA, F. S. R.. Propriedade intelectual: levantamento de patentes no setor de turismo. Revista Brasileira de Administração Científica, v.12, n.2, p.312-320, 2021. DOI: http://doi.org/10.6008/CBPC2179$\underline{684 X .2021 .002 .0025}$ 


\section{INTRODUÇÃO}

As organizações de turismo, nos últimos anos, começaram a investir cada vez mais na gestão do conhecimento, mas ainda é possível perceber a relutância de algumas organizações em empregar a inovação na gestão organizacional das empresas (PIKKEMAAT et al., 2019; RAISI et al., 2020; SOUTO, 2015). A inovação é um fator indispensável no setor de turismo, pois traz uma importante vantagem competitiva para as organizações que aplicam o conhecimento e a informação em seu modelo de negócio, além de ser uma estratégia importante para o crescimento do setor em longo prazo.

As inovações no turismo podem ser tecnológicas e não tecnológicas, sendo que as inovações tecnológicas representam uma pequena porcentagem das inovações geradas no setor de serviços. A inovação neste setor só é considerada bem-sucedida quando os novos conhecimentos aplicados conseguem atender as necessidades dos clientes (SOUTO, 2015).

Embora a proteção de produtos e processos possa ser realizada através do depósito de patentes, é percebível que no setor de turismo as novas ideias são facilmente divulgadas e amplamente aplicadas, inclusive por concorrentes do setor (KOFLER et al., 2018). Este trabalho teve como objetivo analisar, através de uma prospecção tecnológica, o depósito de patentes do setor de turismo registradas no WIPO até novembro de 2019.

\section{REVISÃO TEÓRICA}

\section{Indústria do turismo no Brasil}

O turismo no Brasil é uma atividade econômica relativamente importante, com grande potencial de crescimento futuro. Em 2018, a contribuição do turismo ao PIB brasileiro foi de US\$ 152,5 bilhões (8,1\%), sendo que o PIB turístico teve crescimento de 3,1\%, um dos mais elevados da América do Sul, representando o dobro do que cresceu a economia brasileira. A boa nova do aumento do gasto internacional é atribuída ao recém-criado visto eletrônico oferecido aos Estados Unidos, Canadá, Austrália e Japão.

Segundo dados do World Travel \& Tourism Council's, em 2018 o turismo gerou 6,9 milhões de empregos (7,5\% do total de empregos gerados no Brasil); US\$ 6,2 bilhões aportados em 2018 pelos gastos de estrangeiros, $12,8 \%$ de alta em relação a 2017; lazer, 88\%; e negócios, $12 \%$.

No início do ano de 2019 foram vendidos mais de 275 mil ingressos para torcedores de 83 países de todos os continentes que vieram assistir à 46a edição da Copa América de Futebol (entre 14 de junho e 7 de julho). Ressaltando o potencial turístico das cinco cidades-sede, o diretor de Operações do Comitê Organizador Local da Conmebol Copa América Brasil 2019, Agberto Guimarães, afirma que 'Essa combinação de turismo e futebol, com certeza, é um dos fatores que têm contribuído para a expressiva venda de ingressos até o momento'.

A presidente e CEO do WTTC, Gloria Guevara, afirmou que 'O Brasil ocupa posições de liderança global na contribuição do Turismo para a economia, sendo o aporte do setor para o PIB nacional de $8,1 \%$, gerando 7,5\% dos empregos do país'. 
O Plano Nacional Estratégico de Estatísticas Turísticas brasileiro busca definir diretrizes sobre estatísticas do turismo no Brasil, sob orientação da Organização Mundial do Turismo (OMT), a fim de harmonizar pesquisas do Projeto ConeSul, do qual fazem parte a Argentina, Brasil, Chile, Paraguai e Uruguai.

A Figura 1, apresentada abaixo, traz um resumo de estatísticas e indicadores de turismo no Brasil entre 2016 e 2018.

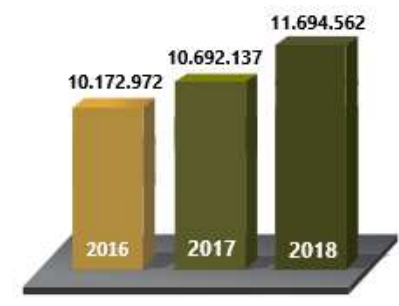

Desembarques Internacionais

Desembarque Internacional de Passageiros - Jan a Dez 2016 a 2018

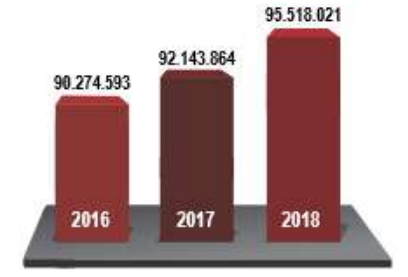

Desembarques Domésticos

Desembarque Nacional de

Passageiros - Jan a Dez 2016 a 2018

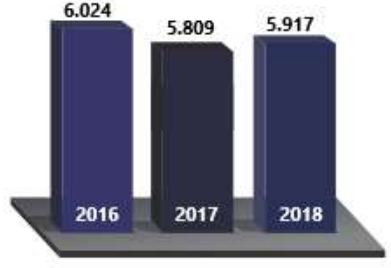

Receita Cambial

Gastos de turistas no Brasil - ( US\$ milhões) - Jan a Dez 2016 a 2018

Figura 1: Desembarques e receita.

O potencial do Brasil para o turismo é desperdiçado em razão de problemas estruturais, sendo um dos países do mundo mais rico em patrimônios naturais e biodiversidade, contudo não há um aproveitamento deste potencial turístico, além disso o país ainda encontra problemas em outras áreas importantes para o desenvolvimento do turismo nacional, como, por exemplo, a falta de segurança, infraestrutura e gestão (BENI, 2019; KIYOTANI, 2019).

A Argentina é o principal emissor de turistas, com 2,5 milhões chegadas, seguido pelos EUA, com 539, 5 mil, Chile, com 387 mil, Paraguai, com 356 mil, Uruguai com 348 mil e França com 238, 3 mil. Apesar da oscilação econômica da Argentina, sua participação no fluxo de turistas internacionais no Brasil continua na liderança desde 2002.

A importância do setor de turismo no Brasil se torna mais aparente quando se avalia seu impacto social, principalmente na geração de empregos. Segundo da Confederação Nacional do Comércio de Bens, Serviços e Turismo (CNC), em 2019 o setor do turismo criou de 35.692 novos postos de trabalho, 163,6\% em relação a 2018. Ainda, segundo a CNC, o setor movimentou em 2019 cerca de $\mathrm{R} \$ 238,6$ bilhões, 2,2\% a mais que em 2018

\section{Inovação no setor de turismo}

As inovações no setor de turismo são de vital importância para manter o setor competitivo, lucrativo e dinâmico e têm como características principais ocorrerem de maneira rápida e contínua. A maioria das inovações no setor turístico são de cunho não tecnológico, pois englobam principalmente mudanças comportamentais e de experiência dos visitantes (PEREIRA et al., 2014; SACRAMENTO et al., 2014).

Dentre as inovações encontradas neste setor atualmente estão o desenvolvimento e utilização de sistemas de informação, a utilização de ferramentas digitais e ferramentas de marketing, as quais auxiliam na rápida disseminação de dados, nas tomadas de decisões pelos empresários, o aumento da produtividade e o suporte para elaboração de estratégias e vantagens competitivas. Além disso, também é possível notar 
mudanças em processos e nos modelos de gestão aplicados no setor (PEREIRA et al., 2014; SACRAMENTO et al., 2014).

\section{Propriedade Intelectual}

De acordo com a Convenção da Organização Mundial da Propriedade Intelectual (WIPO, 2019), a Propriedade Intelectual abrange um amplo espectro, que inclui: Propriedade Industrial - marcas, patentes, desenhos industriais e indicações geográficas utilizadas no meio empresarial; Direito autoral - trabalhos artísticos como livros, pinturas, músicas, filmes, poesias etc.; e a Proteção sui generis - que engloba obras como a topografia de circuito integrado, os cultivares e o conhecimento tradicional.

O manual da WIPO (2019) exemplifica amplamente a Propriedade Intelectual, incluindo obras literárias, artísticas e científicas; performances de artistas, fonogramas e transmissões; invenções em todos os campos do esforço humano; descobertas científicas; desenhos industriais; marcas comerciais, marcas de serviço e nomes e designações comerciais; proteção contra concorrência desleal; e todos os outros direitos resultantes de atividade intelectual na área industrial e científica e no campo literário ou artístico.

A proteção da Propriedade Intelectual é contemplada na legislação, que trata dos direitos que protegem os interesses dos inovadores e criadores, dando-lhes direitos sobre as próprias criações. As nações têm leis para garantir os direitos de criadores e inovadores e promover criatividade e inovação, contribuindo assim para o desenvolvimento econômico e social.

\section{METODOLOGIA}

O estudo exploratório foi realizado com base nos pedidos de patente no setor de turismo depositados na plataforma Organização Mundial de Propriedade Intelectual (em inglês - World Intellectual Property Organization - WIPO). A Organização Mundial de Propriedade Intelectual (OMPI) é uma instituição que contém informações acerca da propriedade intelectual em nível mundial. A pesquisa foi realizada no mês de novembro de 2019.

Foram utilizadas como palavra-chave o termo 'turismo' em português e 'tourism' em inglês. A busca com o termo em português possibilitou encontrar 506 registros de depósitos e com o termo em inglês resultou 10.303 registros de depósitos. Mas quando se utilizou ambos os termos usando o operador booleano 'OR' foram encontrados 14.431 registros de depósitos de patentes para o setor de turismo. Portanto, optou-se em utilizar para este estudo os resultados da busca com os termos 'turismo or tourism' até novembro de 2019, independente da data de depósito.

\section{RESULTADOS E DISCUSSÃO}

A busca de patentes do setor de turismo registradas no WIPO até novembro de 2019 identificou 14.431 depósitos como podemos observar na Tabela 1. 
Tabela 1: Países depositantes $(10+)$ de pedidos de patentes.

\begin{tabular}{l|l|l}
\hline Países & $\mathbf{f i}^{1}$ & $\%$ \\
\hline China & 4.062 & $29 \%$ \\
\hline Estados Unidos da América & 3.573 & $26 \%$ \\
\hline Espanha & 2.869 & $21 \%$ \\
\hline PCT $^{2}$ & 1.330 & $10 \%$ \\
\hline Instituto Europeu de Patentes (IEP) & 485 & $3 \%$ \\
\hline Austrália & 380 & $3 \%$ \\
\hline Brasil & 313 & $2 \%$ \\
\hline República da Coreia & 288 & $2 \%$ \\
\hline Canadá & 283 & $2 \%$ \\
\hline Itália & 213 & $2 \%$ \\
\hline Total & $\mathbf{1 3 . 7 9 6}$ & $\mathbf{1 0 0 \%}$ \\
\hline
\end{tabular}

É mostrado acima os dez países que mais realizarem depósito de pedidos de patentes durante o período analisado, tendo a China como o país que mais depositou pedidos de patentes, seguido dos Estados Unidos, Espanha, Tratado de cooperação de patentes (PCT), Instituto Europeu de patentes (IEP), Austrália, Brasil, República da Coreia, Canadá e Itália.

O Brasil se mostrou na sétima posição no ranking dos dez países que mais depositaram patentes até a data da busca (novembro de 2019), ficando à frente de países como Coreia, Canadá e Itália. A média desse conjunto de dados apresentou um valor de 1.380 patentes depositadas, ou seja, no período em análise, para os dez países que mais depositaram patentes, esse valor representa uma estimativa de quantos registros foram encontrados. A figura 2 mostra a frequência relativa absoluta desse ranking nos países em análise.

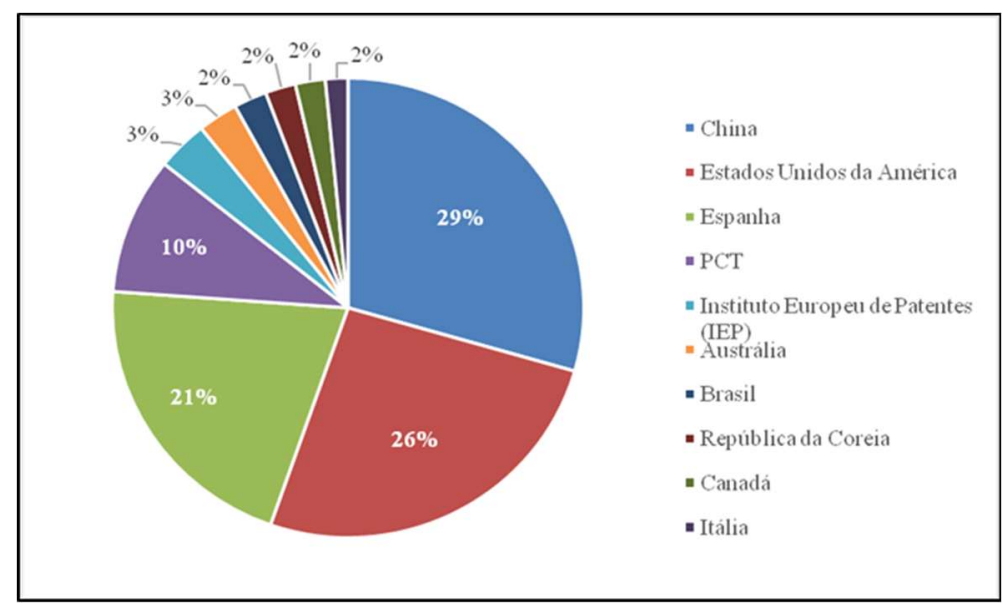

Figura 2: Países depositantes \% (10+).

Percebe-se que quatro países, dentre eles o Brasil, apresentam um percentual similar no número de patentes depositados no período em análise. Esse fato pode ser justificado pelo critério de aproximação, ou seja, o número de registros quantitativo é tão próximo que em média possuem $2 \%$ de pedidos com base no total encontrado em forma percentual. Analisando as principais instituições depositantes de patentes no setor de turismo é possível observar na Tabela 2 as 10 instituições com mais depósitos.

Tabela 2: Requerentes $(10+)$

\begin{tabular}{l|l|l}
\hline Requerentes & $\mathbf{f i}$ & $\%$ \\
\hline Bridgestone corporation & 118 & $17 \%$ \\
\hline Google inc. & 94 & $14 \%$ \\
\hline
\end{tabular}




\begin{tabular}{l|l|l}
\hline $\begin{array}{l}\text { Shandong shengjing tourism } \\
\text { development co., Itd. }\end{array}$ & 92 & $13 \%$ \\
\hline $\begin{array}{l}\text { International business machines } \\
\text { corporation (IBM) }\end{array}$ & 70 & $10 \%$ \\
\hline $\begin{array}{l}\text { Michelin recherche et technique s.a. } \\
\text { Daimlerchrysler ag werke }\end{array}$ & 54 & $10 \%$ \\
\hline $\begin{array}{l}\text { Bayerische tourism } \\
\text { aktiengesellschaft }\end{array}$ & 47 & $9 \%$ \\
\hline $\begin{array}{l}\text { Shandong shengwei } \\
\text { development co., Itd. }\end{array}$ & $\mathbf{8 \%}$ \\
\hline $\begin{array}{l}\text { Wanda cultural tourism planning \& } \\
\text { research institute co., Itd. }\end{array}$ & 42 & $\mathbf{7 \%}$ \\
\hline $\begin{array}{l}\text { Espanola automoviles turismo } \\
\text { Total }\end{array}$ & $\mathbf{6 8 8}$ & $6 \%$ \\
\hline
\end{tabular}

A Tabela acima mostra que a empresa com maior número de depósitos obteve 118 registros e a que menos depositou obteve 41. Se analisarmos cada uma, vemos que não é um número tão expressivo, visto que a média de depósitos foi de 65 patentes se concentrando em torno da mediana, uma vez que esta medida é dada pela soma dos dois valores centrais cujo seu resultado deve ser dividido pelo número 2. Logo, registrase uma mediana de 65 patentes, o que comprova que valores da mediana em torno da média são bem representativos em resultados de pesquisas.

A Figura 3 mostra a representação gráfica em ordem crescente das dez instituições que mais depositaram patentes no setor de turismo no período em análise com base na busca realizada na base de dados em questão.

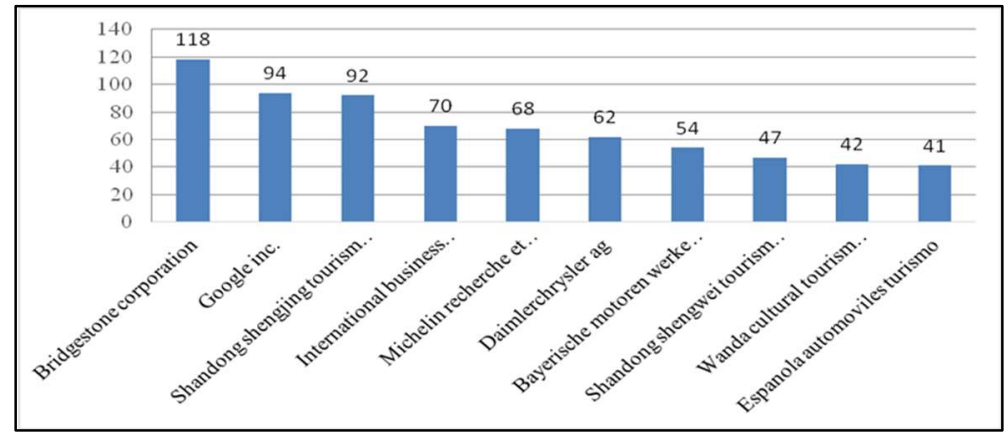

Figura 3: Requerentes (10+).

Pela figura acima, merece destaque a empresa japonesa Bridgestone corporation pois esta foi a empresa que mais depositou patentes no setor do turismo, ficando à frente da Google inc. e da empresa chinesa Shandong shengjing tourism development. Convém chamar atenção para o fato de que das dez empresas que mais depositaram patentes, nenhuma é brasileira, sendo a maioria empresas sediadas nos Estados Unidos, Japão e China.

Em relação ao ano de publicação das patentes, a Tabela 3 mostra o quantitativo de pedidos de patentes do setor de turismo por ano durante o período analisado.

Tabela 3: Ano de publicação das patentes (10+)

\begin{tabular}{l|l|l}
\hline Ano de publicação & fi & $\%$ \\
\hline 2010 & 483 & $5 \%$ \\
\hline 2011 & 525 & $6 \%$ \\
\hline 2012 & 571 & $6 \%$ \\
\hline 2013 & 523 & $6 \%$ \\
\hline
\end{tabular}




\begin{tabular}{l|l|l}
\hline 2014 & 670 & $7 \%$ \\
\hline 2015 & 782 & $9 \%$ \\
\hline 2016 & 883 & $10 \%$ \\
\hline 2017 & 1.357 & $15 \%$ \\
\hline 2018 & 1.972 & $22 \%$ \\
\hline Total & 1.251 & $14 \%$ \\
\hline
\end{tabular}

Pela tabela acima podemos notar que no ano de 2018 ocorreu o maior número de publicações de patentes e 2010 o menor, sendo esses números representando uma porcentagem equivalente a $22 \%$ e $5 \%$ publicações, respectivamente. Abaixo segue a figura 4 mostrando o crescimento e decrescimento desses valores anualmente.

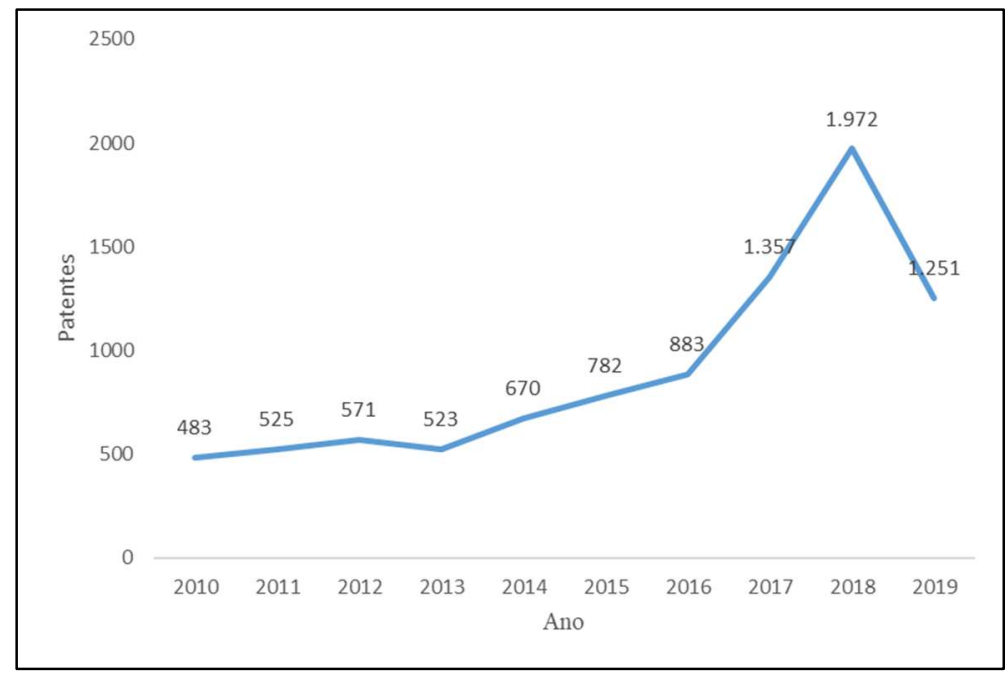

Figura 4: Ano de publicação das patentes (10+).

Analisando a Figura 4, é notório que houve um crescimento significativo nos registros de patentes em questão. $O$ decréscimo que houve no ano de 2013 quando 48 patentes a menos que em 2012 foram depositadas teve esse número aumentado consideravelmente nos anos seguintes, decrescendo novamente em 2019. Esse fato atual pode ser justificado pelo mapeamento da pesquisa, uma vez que no ano em questão só foram realizadas busca de registros até o mês de novembro. Logo, esse número pode ter sido maior para o ano, podendo chegar próximo ao de 2018. A tabela 4 mostra os registros descritivos da variação e desvio referente as patentes, assim como outras medidas.

Tabela 4: Estatística Descritiva.

\begin{tabular}{|c|c|c|c|c|c|c|c|c|}
\hline Valor $\mathrm{n}$ & Média & Mediana & Moda & Mín & Máx & Var & Desvio P. & Coef. Var \\
\hline 9.017 & 901,7 & 726 & Amodal & 483 & 1972 & 234800,2 & 484,5619 & 53,7387 \\
\hline
\end{tabular}

Pela tabela 4, for registrada uma média de 901,7 patentes, com um valor mínimo e máximo de 483 e de 1972 patentes, respectivamente. A mediana frequente nesse conjunto de dados resultou em um número de 726 patentes para o período em análise e a série de dados não apresentou um valor modal, ou seja, não existiu nenhum ano com o mesmo número de patentes, sendo está classificada como amodal. A variação dos dados foi de 234800,2 patentes e desvio de 484,56 patentes. O coeficiente de variação do número de patentes foi de $53,74 \%$ demonstrando uma alta variação nos dados.

Quanto aos códigos mais solicitados nos processos de depósitos de patentes no setor de turismo, 
podemos notar pela Tabela 5 que os resultados descritos possuem uma frequência relativa significativa para os códigos G06F e G06Q com 28\% e 27\% respectivamente.

Tabela 5: Número de patentes por Códigos (10+).

\begin{tabular}{l|l|l}
\hline Código & $\mathbf{f i}$ & $\%$ \\
\hline G06F & 2.366 & $28 \%$ \\
\hline G06Q & 2.294 & $27 \%$ \\
\hline H04L & 652 & $8 \%$ \\
\hline H04N & 581 & $7 \%$ \\
\hline H04W & 539 & $6 \%$ \\
\hline B60R & 457 & $5 \%$ \\
\hline G09F & 415 & $5 \%$ \\
\hline B06K & 414 & $5 \%$ \\
\hline G06T & 401 & $5 \%$ \\
\hline Total & 383 & $5 \%$ \\
\hline & $\mathbf{8 . 5 0 2}$ & $\mathbf{1 0 0 \%}$ \\
\hline
\end{tabular}

O Processamento elétrico de dados digitais (G06F) foi o que apareceu mais na busca, seguido pelo Sistema ou métodos de processamento de dados, especialmente adaptados para propósitos administrativos, comerciais, financeiros de gerenciamento, supervisão ou predição, não incluídos em outro local (G06Q) e Transmissão de informação digital (H04L). Podemos perceber pelos códigos que os depósitos de patentes no setor de turismo estão voltados a produtos tecnológicos de informação e comunicação para gestão. Já o código com menos pedido, no resumo dos 10 +, foi o Processamento de dados de imagem ou geração, em geral (G06T). A figura 5 mostra o comportamento dessa série de dados explicada acima.

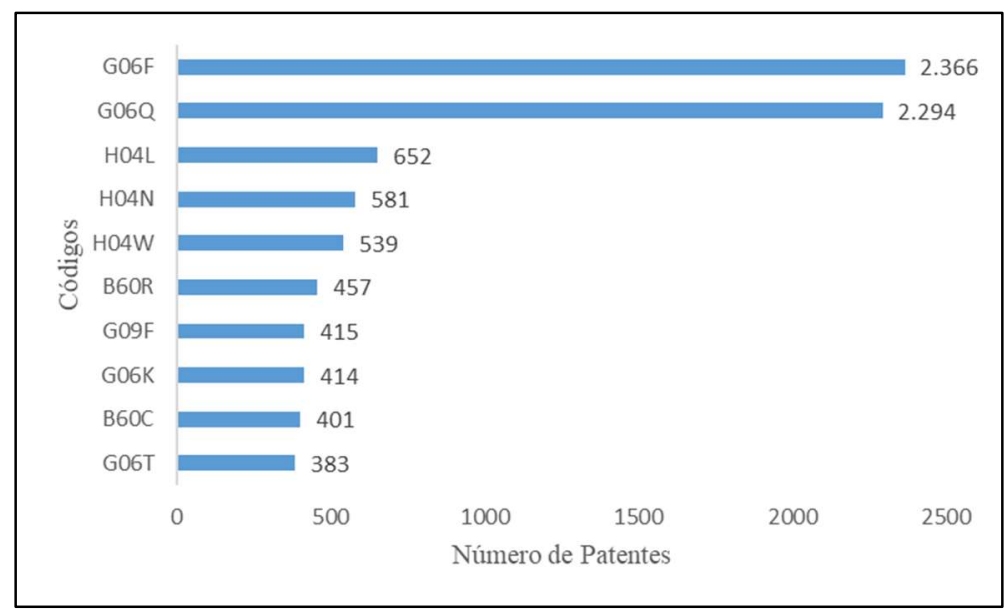

Figura 5: Número de patentes por Códigos (10+).

A Figura 5 mostra que o código G06F e o código G06Q apresentam os maiores números da série de dados, sendo estes 2.366 e 2.294 respectivamente. É fato que existe uma diferença bastante significativa entre os dois códigos iniciais na Figura 5 se compararmos os demais. Os demais registros ficaram com valores em torno de 383 e 652 patentes por códigos.

Apesar desta série apresentar uma média de 850 registros com base no total encontrado, essa diferença para os demais registros reflete na mediana, ou seja, esta medida apresenta um valor de 498 patentes que é um resultado bem disperso com relação à média. 


\section{CONCLUSÕES}

Com o estudo foi possível observar que existe um número expressivo de pedidos e publicações de patentes no setor de turismo, principalmente em termos de processamento elétrico de dados digitais, sistema ou métodos de processamento de dados, especialmente adaptados para propósitos administrativos, comerciais, financeiros de gerenciamento, supervisão ou predição, não incluídos em outro local e transmissão de informação digital.

Observa-se ainda que o Brasil está engatinhando em novas tecnologias para o setor de turismo, estando muito atrás de países como China, Estados Unidos e Espanha, que juntos depositaram 76\% das patentes do setor de turismo.

É possível verificar que o número de publicação de patentes nos três últimos anos (2017 a Nov/2019) com 4.580, superou o número de publicações nos anos de 2010 a 2016 com 4.437. Esses dados demonstram que o setor vem se adaptando aos novos tempos de inovação e tecnologias, e que o futuro é digital.

\section{REFERÊNCIAS}

BRASIL. Lei n. 9.279, de 14 de maio de 1996. Regula direitos e obrigações relativos à Propriedade Industrial. Brasília: DOU, 1996.

BENI, M. C.. Análise estrutural do turismo. Senac, 2019.

KOFLER, I.; MARCHER, A.; VOLGGER, M.; PECHLANER, H.. The special characteristics of tourism innovation networks: The case of the Regional Innovation System in South Tyrol. Journal of Hospitality and Tourism Management, v.37, p.68-75, 2018.

KIYOTANI, I. B.. De região problema à periferia do prazer: o turismo e a ressignificação do Nordeste brasileiro. 2019.

PEREIRA, U. N. C.; OLIVEIRA, E. A. A. Q.. A importância das inovações tecnológicas no setor do turismo. In: CONGRESSO INTERNACIONAL DE CIÊNCIA, TECNOLOGIA E

DESENVOLVIMENTO, 3. Anais. 2014.
PIKKEMAAT, B.; PETERS, M.; BICHLER, B. F.. Innovation research in tourism: Research streams and actions for the future. Journal of Hospitality and Tourism Management, v.41, p.184-196, 2019.

RAISI, H.; BAGGIO, R.; BARRATT-PUGH, L.; WILLSON, G.. A network perspective of knowledge transfer in tourism. Annals of Tourism Research, v.80, p.102817, 2020.

SACRAMENTO, P. M.; TEIXEIRA, R. M.. Adoção de inovações em empresas de pequeno e médio portes: estudo de casos múltiplos em negócios hoteleiros na cidade de Aracaju. Revista Organizações em Contexto, v.10, n.19, 2014.

SOUTO, J. E.. Business model innovation and business concept innovation as the context of incremental innovation and radical innovation. Tourism Management, v.51, p.142155, 2015.

WIPO. Organização Mundial da Propriedade Intelectual. Understanding Industrial Property. WIPO, 2015.

A CBPC - Companhia Brasileira de Produção Científica (CNPJ: 11.221.422/0001-03) detém os direitos materiais desta publicação. Os direitos referem-se à publicação do trabalho em qualquer parte do mundo, incluindo os direitos às renovações, expansões e disseminações da contribuição, bem como outros direitos subsidiários. Todos os trabalhos publicados eletronicamente poderão posteriormente ser publicados em coletâneas impressas sob coordenação da Sustenere Publishing, da Companhia Brasileira de Produção Científica e seus parceiros autorizados. Os (as) autores (as) preservam os direitos autorais, mas não têm permissão para a publicação da contribuição em outro meio, impresso ou digital, em português ou em tradução. 